\title{
Effect of flexible joints on the stability and large deflections of a triangular frame
}

\author{
W. I. Thacker ${ }^{1}$, C.Y. Wang ${ }^{2}$, and L.T. Watson ${ }^{3}$ \\ ${ }^{1}$ Computer Science Department, Winthrop University, Rock Hill, SC 29733, USA \\ ${ }^{2}$ Departments of Mathematics and Mechanical Engineering, Michigan State University, \\ East Lansing, MI 48824, USA \\ ${ }^{3}$ Departments of Computer Science and Mathematics, Virginia Polytechnic Institute and \\ State University, Blacksburg, VA 24061, USA
}

\section{Summary}

An isosceles triangular frame with rotationally resistive joints under a tip load is studied. The large in-plane deformation elastica equations are formulated. Stability analysis shows the frame can buckle symmetrically or asymmetrically. Post-buckling behavior showing limit load and hysteresis are obtained by shooting and homotopy numerical algorithms. The behavior of a frame with rigid joints is studied in detail. The effects of joint spring constant and base length are found.

\section{Introduction}

For traditional structures, only small deflections are tolerated before the material fails. However, some structures are very flexible and return to their original shapes without damage when the disturbance is removed. Examples include springs, linkages, switches, antennas, fishing and vaulting poles, etc. Flexible structures are also desirable in space technology because they are lightweight, can be packed in a tight space and later fully deployed.

If the structural member is thin enough, an accurate constitutive relation can be described by the elastica theory [1-3], where the local moment $m$ is proportional to the local curvature:

$$
m=E I \frac{d \theta}{d s^{\prime}} .
$$

Here $\theta$ is the local angle of inclination, $s^{\prime}$ is the arc length of the member, and $E I$ is the flexural rigidity. The equilibrium shape of an elastica can then be described by a set of nonlinear differential equations, which can be integrated numerically or expressed in terms of elliptic functions. We shall not discuss the large deflections of simple members such as the cantilever under a tip load or the pulling apart of circular and square rings, where the deformation behaviors are well understood (see e.g., [3-6]).

In the eccentric loading of a portal frame it was found that the limit load may be smaller than the bifurcation load, making the system imperfection sensitive [7-9]. The buckling and truly large deformation (up to snap through) of a portal frame was studied by Huddleston [10]. Square and gabled frames, although for small deformations, were also analyzed [11-14].

Thacker et al. [15, 16] considered a rigid platform whose weight is supported by elastica legs. Unlike eccentric loading, the buckling load is finite and can be predicted by 
linear bifurcation analysis. There is also a global buckling load (limit load), which is lower than the buckling load. Below the global buckling load, the flexible structure would return to its original shape after the removal of any imposed disturbance.

All of the above sources considered rigidly connected joints. The most basic frame with hinged joints is the isosceles triangular frame studied by Wang [17]. Using an analytical expression for the force-displacement relation of a single bar [18], Wang was able to delineate the complete post-buckling properties of the hinged flexible triangular frame, including snap-through. It was found that there is a single linear buckling load, but unlike all previous literature on rigid frames, the post-buckling picture is quite complicated. There exist distinct bifurcation curves when the legs of the triangular frame are both curved and symmetric, both curved and asymmetric, or one straight and one curved. Again unlike rigid frames, the hinged frame is always unstable for some finite disturbance, thus there is no global buckling load. Notice a rectangular frame with all hinged joints would not have any structural rigidity.

Structures with flexible joints are also practical and important. Deployable space structures have rotational spring joints. On the other hand, the joints of terrestrial structures may not be entirely rigid. For a rectangular frame, the effect of flexible joints lowers the buckling load, and its immediate post-buckling was also reported [19-21].

The triangular frame would behave differently than a rectangular frame. It would be interesting to see how the properties of a triangular frame with rigid joints evolve to that with hinged joints. The aim of the present paper is to consider the triangular frame with rotationally flexible joints. The complicated post-buckling behavior is presented here for the first time.

\section{Formulation}

Fig. 1a shows an isosceles triangular frame with two legs of equal length $L$ and the base length $b L$. The joints are modeled by rotational hinges with spring constant $k^{\prime}$. A vertical force $F^{\prime}$ acts on the tip of the frame. If the frame buckles, the deformed shape is shown in Fig. 1b. Let the left leg be denoted by the subscript 1 and the right by the subscript 2 . The origin of Cartesian axes $\left(y^{\prime}, x^{\prime}\right)$ is located at the base of the left leg where the vertical and horizontal forces are $F_{1}^{\prime}$ and $G^{\prime}$ as shown. For the right leg, the base reactions are $F_{2}{ }^{\prime}$ and $G^{\prime}$. For equilibrium, $F^{\prime}=F_{1}{ }^{\prime}+F_{2}{ }^{\prime}$. There are also external moments acting at the bases. Considering the equilibrium of an elemental length of the left leg (Fig. 1c), the moment balance gives

$$
m+d m+F_{1}{ }^{\prime} \sin \theta_{1} d s^{\prime}=m+G^{\prime} \cos \theta_{1} d s^{\prime} .
$$

Normalize the length by $L$, the forces by $E I / L^{2}$ and drop primes. Eqs. $(1,2)$ then give the elastica equation

$$
\frac{d^{2} \theta_{1}}{d s^{2}}+F_{1} \sin \theta_{1}-G \cos \theta_{1}=0 .
$$

Similarly for the right leg

$$
\frac{d^{2} \theta_{2}}{d s^{2}}+F_{2} \sin \theta_{2}+G \cos \theta_{2}=0 \text {. }
$$

The kinematic conditions are 


$$
\frac{d x_{1}}{d s}=\cos \theta_{1}, \quad \frac{d y_{1}}{d s}=\sin \theta_{1}, \quad \frac{d x_{2}}{d s}=\cos \theta_{2}, \quad \frac{d y_{2}}{d s}=\sin \theta_{2} .
$$

The boundary conditions at the base are

$$
x_{1}(0)=0, \quad y_{1}(0)=0, \quad x_{2}(0)=0, \quad y_{2}(0)=b .
$$

Continuity at the top gives

$$
x_{1}(1)=x_{2}(1), \quad y_{1}(1)=y_{2}(1) .
$$

In the undeformed state, both legs are straight with the inclination angles

$$
\theta_{1}=-\theta_{2}=\sin ^{-1}(b / 2) \equiv \theta_{0} \text {. }
$$

At the left base (origin), the moment is proportional to the angle difference:

$$
M_{1}=E I \frac{d \theta_{1}}{d s^{\prime}}(0)=k^{\prime}\left[\theta_{1}(0)-\theta_{0}\right]
$$

or

$$
\frac{d \theta_{1}}{d s}(0)=k\left[\theta_{1}(0)-\theta_{0}\right]
$$

where $k=k^{\prime} L / E I$ is the normalized rotational spring constant. Similarly at the right base

$$
\frac{d \theta_{2}}{d s}(0)=k\left[\theta_{2}(0)+\theta_{0}\right]
$$

On the top vertex, the flexible joint condition gives

$$
\frac{d \theta_{1}}{d s}(1)=k\left[2 \theta_{0}-\theta_{1}(1)+\theta_{2}(1)\right]
$$

A global moment balance about the origin yields

$$
F y_{1}(1)=\frac{d \theta_{1}}{d s}(0)+\frac{d \theta_{2}}{d s}(0)+F_{2} b \text {. }
$$

Given the total load $F=F_{1}+F_{2}$, the spread $b$, and the spring constant $k$, Eqs. (3-5) are eight first order equations plus two unknowns $\left(F_{2}, G\right)$. There are ten boundary conditions Eqs. (6, 7, 10-13). The problem to be solved is highly nonlinear. We note that when $k=0$ all joints are freely hinged and when $k=\infty$ all joints are rigid.

\section{Stability analysis}

The undeformed state is symmetric (unlike imperfection problems). The vertical and horizontal forces in each leg are

$$
F_{0}=\frac{F}{2}, \quad G_{0}=\frac{F}{2} \tan \theta_{0},
$$

where $\theta_{0}$ is defined in Eq. (8). We perturb from this state as follows:

$$
\begin{aligned}
& F_{1}=F_{0}+f_{1}, \quad F_{2}=F_{0}+f_{2}, \quad G=G_{0}+g, \\
& \theta_{1}=\theta_{0}+\varphi_{1}, \quad \theta_{2}=-\theta_{0}+\varphi_{2}, \\
& x_{1}=s \cos \theta_{1}+\xi_{1}, \quad y_{1}=s \sin \theta_{1}+\eta_{1}, \\
& x_{2}=s \cos \theta_{2}+\xi_{2}, \quad y_{2}=b-s \sin \theta_{2}+\eta_{2} .
\end{aligned}
$$

Here the last terms in Eqs. $(15,16)$ are much smaller than unity. Since the total force is $F$, 


$$
f_{1}+f_{2}=0 .
$$

Upon substitution into Eqs. (3-5), we find the linearized equations are

$$
\begin{aligned}
& \frac{d^{2} \varphi_{1}}{d s^{2}}+F_{0} \cos \theta_{0} \varphi_{1}+\sin \theta_{0} f_{1}+G_{0} \sin \theta_{0} \varphi_{1}-\cos \theta_{0} g=0, \\
& \frac{d^{2} \varphi_{2}}{d s^{2}}+F_{0} \cos \theta_{0} \varphi_{2}-\sin \theta_{0} f_{2}+G_{0} \sin \theta_{0} \varphi_{2}+\cos \theta_{0} g=0, \\
& \frac{d \xi_{1}}{d s}=-\sin \theta_{0} \varphi_{1}, \quad \frac{d \eta_{1}}{d s}=\cos \theta_{0} \varphi_{1}, \\
& \frac{d \xi_{2}}{d s}=\sin \theta_{0} \varphi_{2}, \quad \frac{d \eta_{2}}{d s}=\cos \theta_{0} \varphi_{2} .
\end{aligned}
$$

The boundary conditions Eqs. $(6,7,10-13)$ become

$$
\begin{aligned}
& \xi_{1}(0)=\eta_{1}(0)=0, \quad \xi_{2}(0)=\eta_{2}(0)=0, \\
& \xi_{1}(1)=\xi_{2}(1), \quad \eta_{1}(1)=\eta_{2}(1), \\
& \frac{d \varphi_{1}}{d s}(0)=k \varphi_{1}(0), \quad \frac{d \varphi_{2}}{d s}(0)=k \varphi_{2}(0), \\
& \frac{d \varphi_{1}}{d s}(1)=k\left[\varphi_{2}(1)-\varphi_{1}(1)\right], \\
& F \eta_{1}(1)=\frac{d \varphi_{1}}{d s}(0)+\frac{d \varphi_{2}}{d s}(0)+b f_{2} .
\end{aligned}
$$

The nontrivial trivial solution to Eqs. (18-31) determines the buckling load. We distinguish between the symmetric case and the asymmetric case.

\section{(A) Symmetric case}

In this case, the frame deforms symmetrically and we can consider only the left leg. Since $\eta_{1}=-\eta_{2}, \varphi_{2}=-\varphi_{1}$ and $f_{2}=f_{1}=0$, Eqs. $(27,30)$ become

$$
\eta_{1}(1)=0, \quad \frac{d \varphi_{1}}{d s}(1)=-2 k \varphi_{1}(1) .
$$

Eq. (18) is then integrated to give

$$
\varphi_{1}=C_{1} \sin (A s)+C_{2} \cos (A s)+g \cos \theta_{0} / A^{2},
$$

where $C_{1}$ and $C_{2}$ are constants and

$$
A^{2}=F_{0} \cos \theta_{0}+G_{0} \sin \theta_{0}=\frac{F}{2 \cos \theta_{0}} .
$$

Eqs. $(20,21,24)$ then yield

$$
\begin{aligned}
& \xi_{1}=-\frac{\sin \theta_{0}}{A}\left(C_{1}[1-\cos (A s)]+C_{2} \sin (A s)+g \cos \theta_{0} s / A\right), \\
& \eta_{1}=\frac{\cos \theta_{0}}{A}\left(C_{1}[1-\cos (A s)]+C_{2} \sin (A s)+g \cos \theta_{0} s / A\right) .
\end{aligned}
$$

Eqs. $(28,32)$ give 


$$
\begin{aligned}
& A C_{1}-k C_{2}-\frac{k \cos \theta_{0}}{A^{2}} g=0, \\
& (1-\cos A) C_{1}+\sin A C_{2}+\frac{\cos \theta_{0}}{A} g=0,
\end{aligned}
$$

$$
(A \cos A+2 k \sin A) C_{1}-(A \sin A-2 k \cos A) C_{2}+\frac{2 k \cos \theta_{0}}{A^{2}} g=0 .
$$

For nontrivial solutions $C_{1}, C_{2}$, and $g$ of Eq. (37) the determinant of the coefficient matrix of Eq. (37) must be zero. The characteristic equation is

$$
\begin{aligned}
& {\left[A^{2}+k(1-\cos A)\right][A \sin A+2 k(1-\cos A)]} \\
& \quad+k(\sin A-A)[A \cos A+2 k \sin A+2 A]=0 .
\end{aligned}
$$

For a given spring constant $k$, the eigenvalues $A$ can be found from Eq. (38) by a root finder. Eq. (34) then gives the buckling force

$$
F=2 A^{2} \cos \theta_{0}=A^{2} \sqrt{4-b^{2}} .
$$

Table 1 gives the lowest eigenvalue $\mathrm{A}$, which depends only on the spring constant $k$. The symmetric buckling force is proportional to $A^{2}$ and a factor depending on the leg spread $b$ as in Eq. (39).

(B) Asymmetric case

Since $\varphi_{1} \neq-\varphi_{2}$, let

$$
\psi=\varphi_{1}+\varphi_{2}, \quad \chi=\xi_{1}-\xi_{2}, \quad \zeta=\eta_{1}+\eta_{2},
$$

Adding Eqs. $(18,19)$ we have

$$
\frac{d^{2} \psi}{d s^{2}}+\left(F_{0} \cos \theta_{0}+G_{0} \sin \theta_{0}\right) \psi-2 \sin \theta_{0} f_{2}=0 .
$$

The solution is

$$
\psi=C_{3} \sin (A s)+C_{4} \cos (A s)+C_{5},
$$

where $A$ is defined in Eq. (34) and

$$
C_{5}=\frac{2 \sin \theta_{0} f_{2}}{A^{2}} .
$$

Add Eqs. $(28,29)$ and then use Eq. (42) to obtain

$$
A C_{3}=k\left(C_{4}+C_{5}\right) \text {. }
$$

Subtracting Eqs. $(20,22)$ yields

$$
\frac{d \chi}{d s}=-\sin \theta_{0} \psi \text {. }
$$

Using Eqs. $(24,25,42)$ the solution is

$$
\chi=-\frac{\sin \theta_{0}}{A}\left(C_{3}[1-\cos (A s)]+C_{4} \sin (A s)+A C_{5} s\right) .
$$

The boundary condition Eq. (26) gives

$$
(1-\cos A) C_{3}+\sin A C_{4}+A C_{5}=0 .
$$

Adding Eqs. $(21,23)$ gives

$$
\frac{d \zeta}{d s}=\cos \theta_{0} \psi \text {. }
$$


With Eqs. $(24,25)$ the solution is

$$
\zeta=\frac{\cos \theta_{0}}{A}\left(C_{3}[1-\cos (A s)]+C_{4} \sin (A s)+C_{5} A s\right) .
$$

Eqs. $(27,31,43)$ lead to the boundary condition

$$
F \frac{\zeta(1)}{2}=\frac{d \psi}{d s}(0)+\frac{C_{5} b A^{2}}{2 \sin \theta_{0}}
$$

or

$$
\left(\sin ^{2} \theta_{0}+\cos A \cos ^{2} \theta_{0}\right) C_{3}-\sin A \cos ^{2} \theta_{0} C_{4}+A \sin ^{2} \theta_{0} C_{5}=0 .
$$

For nontrivial $C_{3}, C_{4}$ and $C_{5}$, Eqs. $(44,47,51)$ yield a characteristic equation that simplifies to

$$
\left(k+A^{2}\right) \tan A-k A=0 .
$$

After the lowest eigenvalue $A$ is found, Eq. (39) then yields the buckling force. Table 2 shows the values of lowest $A$ for asymmetric buckling.

\section{Numerical Methods}

The boundary value problem for the spring jointed triangle frame is defined by Eqs. (3-5) with boundary conditions $(6,7,10-13)$; to solve this, we need values for $F, F_{1}, k, G, \theta_{1}(0)$ and $\theta_{2}(0)$. Since there are only four boundary conditions at $s=1$, we can only solve for four variables; the rest must be given as parameters of the problem. We use $F$ and $k$ as the problem parameters. The unknowns are $\theta_{1}(0), \theta_{2}(0), G$, and $F_{1}$.

Let

$$
V=\left(\begin{array}{c}
\theta_{1}(0) \\
\theta_{2}(0) \\
G \\
F_{1}
\end{array}\right),
$$

and let $\theta_{1}(s ; V), \theta_{2}(s ; V), \quad x_{1}(s ; V), \quad x_{2}(s ; V), \quad y_{1}(s ; V), \quad y_{2}(s ; V)$ be the solution to the initial value problem given by Eqs. $(3-7,12,13)$ with unknown values specified by $V$. The original two-point boundary value problem is mathematically equivalent to solving the nonlinear system of equations

$$
\Psi(V)=\left(\begin{array}{c}
x_{1}(1 ; V)-x_{2}(1 ; V) \\
y_{1}(1 ; V)-y_{2}(1 ; V) \\
\theta_{1}(1 ; V)-k\left[\theta_{1}(1 ; V)-\theta_{2}(1 ; V)-2 \sin ^{-1}\left(\frac{b}{2}\right)\right] \\
F y_{1}(1 ; V)-\theta_{1}^{\prime}(0 ; V)-\theta_{2}^{\prime}(0 ; V)-F_{2} b
\end{array}\right)=0 .
$$

The software used for solving the initial value problems in the shooting formulation (54) (Keller [22]) was the subroutine ODE from ODEPACK (Shampine and Gordon [23]). (54) is a very difficult nonlinear system of equations. 
This problem has multiple solutions for certain $F$ and $b$ values. The trivial solution $V=\left(\sin ^{-1}(.5 b),-\sin ^{-1}(.5 b),(F / 2) \tan \left(\sin ^{-1}(.5 b)\right), F / 2\right)^{T}$ is a solution for all $F$ and $k$. A quasi-Newton method, implemented in the subroutine HYBRJ from the MINPACK library (Moré, Garbow, and Hillstrom [24]), was used to attempt to solve (54). However, starting points close enough to a nontrivial solution for the quasi-Newton method to converge to the solution could not be found. HYBRJ would either stagnate in a nonzero local minimum of $\Psi^{T} \Psi$ or converge to the trivial solution.

Next, a globally convergent homotopy based nonlinear system solver, subroutine FIXPDF from the HOMPACK (Watson, Billups, and Morgan [25]) suite of codes, was used. This method (using the default homotopy map) tracks the zero curve of

$$
\rho_{a}(\Lambda, V)=\Lambda \Psi(V)+(1-\Lambda)(V-a),
$$

starting with $\Lambda=0 . \rho_{a}(0, V)=0$ when $V=a$, so a solution is known for $\rho_{a}(\Lambda, V)=0$ when $\Lambda=0$. From this starting point, solutions of $\rho_{a}(\Lambda, V)=0$ are tracked until $\Lambda=1$. The HOMPACK codes track this curve allowing $\Lambda$ to decrease also. This is needed for many nonlinear systems and is not provided with standard continuation.

The homotopy method did not have problems with local minima like the quasiNewton methods. Whereas, FIXPDF always converged, it sometimes converged to the trivial solution. Instead, AUTO (Doedel, et al. [26]), a bifurcation analysis package, was used to find non-trivial solutions. Using a nontrivial solution from AUTO as a starting point to HYBRJ, problems with slightly different $F$ and $k$ were solved. Too large a step size could not be taken in $F$ or $k$ or HYBRJ would not converge. When this would happen, the HOMPACK system would be used to find a solution.

For a fixed $k$, we want to track the zero curve

$$
\Gamma=\left\{(F, V) \mid \Psi(V ; F)=0, \quad F_{0} \leq F \leq F_{m}\right\}
$$

of $\Psi(V ; F)$ as $F$ varies between some $F_{0}$ and $F_{m}$. This would be possible using HYBRJ if the solution $V=V(F)$ along the zero curve $\Gamma$. If $V$ is not a function of $F$ along $\Gamma$, HYBRJ cannot be used to advance the solution from a prior solution $\left(F_{0}, V_{0}\right)$ because $F$ can either increase or decrease along $\Gamma$. HOMPACK is designed to solve such problems, however, and the subroutine STEPS from HOMPACK can be used to trace $\Gamma$ between any two connected solutions $\left(F_{0}, V_{0}\right)$ and $\left(F_{m}, V_{m}\right)$.

The bifurcation diagrams (using vertical displacement of the tip

$$
\delta=\cos \left(\sin ^{-1}(b / 2)\right)-x_{1}(1)
$$

as the dependent variable) were produced using the zero curve results as input to the initial value solver ODE to get $x_{1}(1)$. The frames were plotted by reading $V$ into Mathematica (Wolfram [27]) and having it solve the initial value problem for $x_{1}, y_{1}$ and $x_{2}, y_{2}$, then performing a parametric plot of these functions.

\section{Results and Discussions}

First consider the case when the joints are hinged with no rotational resistance $(k=0)$. Our stability analysis Eqs. $(38,52)$ show both symmetric and asymmetric buckling occur at $A=n \pi$. Thus from Eq. (39) the buckling load is $\pi^{2} \sqrt{4-b^{2}}$. The post-buckling 
behavior is then computed by our numerical method. The tip force-vertical displacement curves for the equilateral triangle $(b=1)$ case are shown in Fig. 2. In essence, infinitesimal buckling begins at the critical force $F=17.095$ (Point A). Symmetric buckling follows the solid curve ABEK and asymmetric (one leg curved, one leg straight) buckling follows the dashed curve AGCH. There is also a secondary both-legs-curved asymmetric branch EJ. These results confirm those of [17], which used a completely different, individual column method.

However, the method used by [17] cannot treat any frame with non-zero joint rotational resistance. Consider the important case of a rigidly joined $(k=\infty)$ frame, which has never been studied. Eq. (38) shows the symmetric bifurcation occurs at the root of

$$
2(1-\cos A)-A \sin A=0
$$

or $A=6.2832$. For the equilateral triangle, the critical force is $F=68.38$. On the other hand, for the asymmetric case, Eq. (52) reduces to $\tan A-A=0$

or $A=4.4934$. The critical force for $b=1$ is $F=34.97$. Fig. 3 shows the complete force-displacement picture. We note there are two disjoint bifurcation curves. The symmetric post-buckling follows ABK while the asymmetric deformation follows LGCHM. Let us increase the tip load on this frame from zero gradually. The first critical load is reached at state $\mathrm{L}(F=34.97)$ but the asymmetric post-buckling is immediately unstable since the slope of the path LG is negative, which indicates the load would be doing negative work. We expect the frame would catastrophically jump to state $\mathrm{C}$ at the same load. As the load is further increased, the deformation would follow the segment $\mathrm{CH}$, which has positive slope. There is no stable equilibrium load beyond state $\mathrm{H}$ $(F=36.26)$ and the frame would snap through to the other side (via HM). However, a hysteresis loop is formed if the load is decreased from state C back to state $\mathrm{G}$ $(F=29.71)$ and then the frame snaps into the undeformed shape. The symmetric mode has a much higher critical load (68.38) at state A and is unlikely to occur when the load is gradually increased. The segment up to state $\mathrm{B}(F=69.16)$ is stable. These predictions do not apply to large perturbations. For example, a load of 32 (between states L and G) when gradually applied would not cause any deformation. However, the same load if adequately perturbed (kicked) may land the frame on the stable segment GC. If overly perturbed, the frame would snap through to the other side. The stable symmetric deformation on $\mathrm{AB}$ can be similarly realized. Figure 4 shows the asymmetric deformed shapes corresponding to the (rigidly joined) states shown in Fig. 3. Note that the states $\mathrm{M}$ and $\mathrm{K}$ are in equilibrium with zero load, but unstable to any perturbation. The shapes of the states $\mathrm{C}, \mathrm{H}, \mathrm{M}$ would bend below the $x$-axis, if not constrained by a solid base line at $x=0$. Figure 5 shows the corresponding symmetric deformed shapes, which require a higher tip load.

The force-displacement curves of a rigidly joined triangular frame for various base lengths are shown in Fig. 6. First, consider symmetric post-buckling. If the base length is zero $(b=0)$, Eqs. $(39,58)$ show the bifurcation occurs at $F=78.957$. This agrees with twice the buckling load of a clamped-clamped column, which is $8 \pi^{2}$. As $b$ is increased, the symmetric post-buckling curves gradually collapse into the origin, occurring at $b=2$. More involved are the asymmetric force-displacement curves. For $b \rightarrow 0$, Eqs. $(39,59)$ give $F=40.381$. However, twice the buckling load of a clamped- 
free column is $F=\pi^{2} / 2=4.935$. The singular behavior as $b \rightarrow 0$ is also reflected by the $b=0.25$ asymmetric post-buckling curve. For larger $b$, the post-buckling curves may have several inflection points, and some are quite complicated. For example, the $b \geq 1$ curves bend into the negative $F$ half plane, but the $b<1$ curves tend toward infinite $F$. However, a discussion of the asymptotic trend is academic, since the tip touches the base line when the displacement reaches $\sqrt{1-b^{2} / 4}$, and parts of the bent legs may have touched the base line even earlier.

The effect of the joint stiffness is shown in Fig. 7 for base length $b=1$. For $k=0$, or pinned joints (Fig. 7(a) or Fig. 2), the force-displacement curves include a symmetric post-buckling curve, an asymmetric post-buckling curve, and a secondary asymmetric post-buckling curve. As the joint stiffness is increased, both buckling loads and post-buckling loads increase, as more energy is required to deform a more constrained system. The secondary asymmetric bifurcation eventually merges with the symmetric post-buckling curve, as depicted in Fig. 7(c). For large $k$, the characteristics of the force-displacement curves can be represented by the rigidly joined case (Fig. 7(d) and Fig. 3).

The effect of narrower or wider base lengths is shown in Figs. 8 and 9. The buckling forces are larger for a smaller base length. However, the asymmetric forcedisplacement curve is quite horizontal for small displacements, making the frame unstable and imperfection sensitive (Fig. 8). On the other hand, when the base length is larger (Fig. 9), all modes have negative slope and are unstable. In comparison, the equilateral triangular frame ( $b=1$, Fig. 7) has the advantage of stability (positive slope segments) if displacements are limited to some range.

In conclusion, we have solved the difficult problem of large elastic deformations of a triangular frame. Nonlinear phenomena such as instability, hysteresis, limit load, snap through, and imperfection sensitivity are found. It is hoped that our methods and results would elicit further research on the large deformations of frames.

\section{References}

[1] Love, A.E.H.: A Treatise on the Mathematical Theory of Elasticity, $4^{\text {th }}$ ed. New York: Dover 1944.

[2] Timoshenko, S.P. and Gere, J.M.: Theory of Elastic Stability, $2^{\text {nd }}$ ed. New York: McGraw 1961.

[3] Frisch-Fay, R.: Flexible Bars, London: Butterworths 1962.

[4] Wang, C.Y.: Large deflections of an inclined cantilever with an end load. Int. J. Nonlinear Mech. 16, 155-164 (1981).

[5] Watson, L.T. and Wang, C.Y.: A homotopy method applied to elastica problems. Int. J. Solids Struct. 17, 29-37 (1981).

[6] Ohtsuki, A. and Ellyin, F.: Large deformation analysis of a square frame with rigid joints. Thin Walled Struct. 38, 79-91 (2000).

[7] Hutchinson, J.W. and Koiter, W.T.: Postbuckling theory. Appl. Mech. Rev. 23, 13531366 (1970).

[8] Simitses, G.J. and Kounadis, A.N.: Buckling of imperfect rigid joined frames. J. Eng. Mech. 104, 569-586 (1978). 
[9] Chistodoulou and Kounadis, A.N.: Elastica buckling analysis of a simple frame. Acta Mech. 61, 153-163 (1986).

[10] Huddleston, J.V.: Nonlinear buckling and snap over of a two-member frame. Int. J. Solids Struct. 3, 1023-1030 (1967).

[11] Brush, D.O. and Almroth, B.O.: Buckling of Bars, Plates and Shells, New York: McGraw 1975.

[12] Simitses, G.J.: An Introduction to the Elastic Stability of Structures, New Jersey: Prentice-Hall, 1976.

[13] Simitses, G.J., Giri, J. and Kounadis, A.N.: Nonlinear analysis of portal frames. Int. J. Num. Meth. Eng. 17, 123-132 (1981).

[14] Qashu, R.K. and DaDeppo, D.A.: Large deflection and stability of rigid frames. J. Eng. Mech. 109, 765-780 (1983).

[15] Thacker, W.I., Wang, C.Y. and Watson, L.T.: The nonlinear stability of a heavy rigid plate supported by flexible columns. Int. J. Solids Struct. 30, 3443-3449 (1993).

[16] Thacker, W.I., Wang, C.Y. and Watson, L.T.: Global stability of a thick solid supported by elastica columns. J. Eng. Mech. 123, 287-289 (1997).

[17] Wang, C.Y.: Analysis of nonlinear deformations of a triangular frame. Mech. Struct. Mach. 28, 237-243 (2000).

[18] Wang, C.Y.: Asymptotic formula for the flexible bar. Mech. Mach. Th. 34, 645-655 (1999).

[19] Romstad, K.M. and Subramanian, C.V.: Analysis of frames with partial connection rigidity. ASCE J. Struct. Div. 96, 2283-2300 (1970).

[20] Lui, E.M. and Chen, W.F.: Analysis and behavior of flexibly-joined frames. Eng. Struct. 8, 107-118 (1986).

[21] Goto, Y., Suzuki, S. and Chen, W.F.: Stability behavior of semirigid sway frames. Eng. Struct. 15, 209-219 (1993).

[22] Keller, H.B.: Numerical Solution of Two-Point Boundary Value Problems, Philadelphia: Society for Industrial and Applied Mathematics, 1976.

[23] Shampine, L.F. and Gordon, M.K.: Computer Solution of Ordinary Differential Equations, San Francisco: W. H. Freeman, 1975.

[24] Moré, J.J., Garbow, B.S. and Hillstrom, K.E.: User Guide for MINPACK-1, ANL80-74, Argonne, IL: Argonne National Laboratory, 1980.

[25] Watson, L.T., Billups, S.C. and Morgan, A.P.: Algorithm 652: HOMPACK: a suite of codes for globally convergent homotopy algorithms, ACM Trans. Math. Software 13, 281-310 (1987).

[26] Doedel, E.J., Champneys, A.R., Fairgrieve, T.F., Kuznetsov, Y.A., Sandstede, B., and Wang, X.: AUTO 97: Continuation and Bifurcation Software for Ordinary Differential Equations with HomCont. User Guide. http://indy.cs.concordia.ca/auto/. (1997).

[27] Wolfram, S.: Mathematica, A System for Doing Mathematics by Computer, Redwood City, CA: Addison-Wesley, 1988. 
Table 1. Lowest eigenvalue $A$ and corresponding bifurcation value $F$ for various constants $k$ (symmetric case).

\begin{tabular}{|l|l|l|l|l|}
\hline$k$ & $A$ & $F(b=0.5)$ & $F(b=1.0)$ & $F(b=1.5)$ \\
\hline 0 & 3.142 & 19.11 & 17.09 & 13.06 \\
\hline 0.02 & 3.161 & 19.34 & 17.30 & 13.21 \\
\hline 0.1 & 3.234 & 20.25 & 18.11 & 13.84 \\
\hline 0.2 & 3.321 & 21.35 & 19.10 & 14.59 \\
\hline 0.5 & 3.552 & 24.43 & 21.85 & 16.69 \\
\hline 1 & 3.863 & 28.90 & 25.85 & 19.74 \\
\hline 2 & 4.311 & 35.99 & 32.19 & 24.59 \\
\hline 5 & 5.024 & 48.88 & 43.72 & 33.39 \\
\hline 10 & 5.510 & 58.78 & 52.58 & 40.16 \\
\hline 100 & 6.190 & 74.21 & 66.37 & 50.69 \\
\hline$\infty$ & 6.283 & 76.45 & 68.38 & 52.23 \\
\hline
\end{tabular}

Table 2. Lowest eigenvalue $A$ and corresponding bifurcation value $F$ for various constants $k$ (asymmetric case).

\begin{tabular}{|l|l|l|l|l|}
\hline$k$ & $A$ & $F(b=0.5)$ & $F(b=1.0)$ & $F(b=1.5)$ \\
\hline 0 & 3.142 & 19.11 & 17.09 & 13.06 \\
\hline 0.02 & 3.148 & 19.15 & 17.16 & 13.11 \\
\hline 0.1 & 3.173 & 19.49 & 17.44 & 13.32 \\
\hline 0.2 & 3.203 & 19.86 & 17.77 & 13.57 \\
\hline 0.5 & 3.286 & 20.91 & 18.70 & 14.28 \\
\hline 1 & 3.406 & 22.46 & 20.09 & 15.34 \\
\hline 2 & 3.909 & 29.58 & 26.46 & 20.21 \\
\hline 5 & 4.132 & 33.07 & 29.58 & 22.59 \\
\hline 10 & 4.449 & 38.34 & 34.29 & 26.19 \\
\hline 100 & 4.489 & 39.02 & 34.90 & 26.66 \\
\hline$\infty$ & 4.493 & 39.10 & 34.97 & 26.71 \\
\hline
\end{tabular}




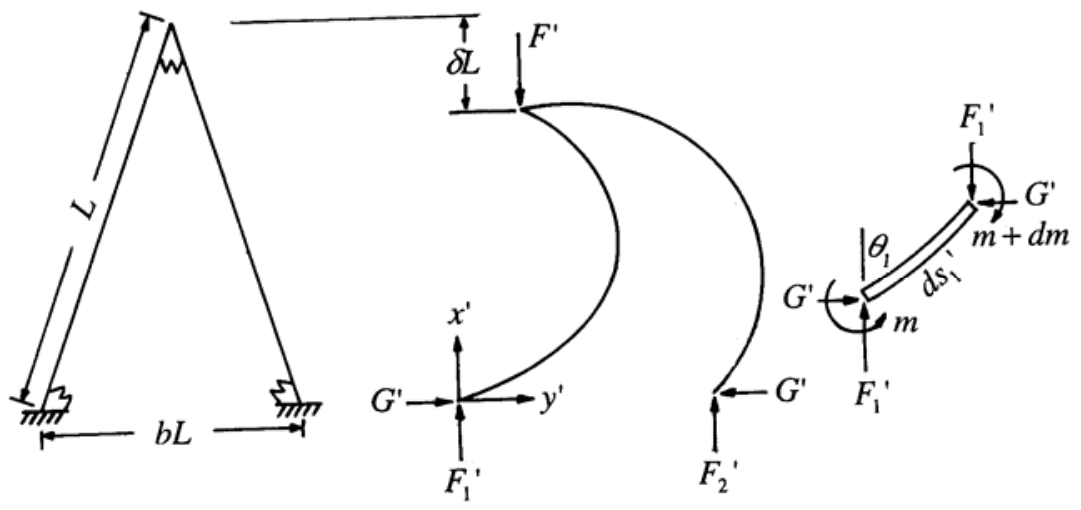

(a)

(b)

(c)

Fig. 1. (a) The undeformed triangular frame (b) Post-buckling under a tip load (c) An elemental length. 


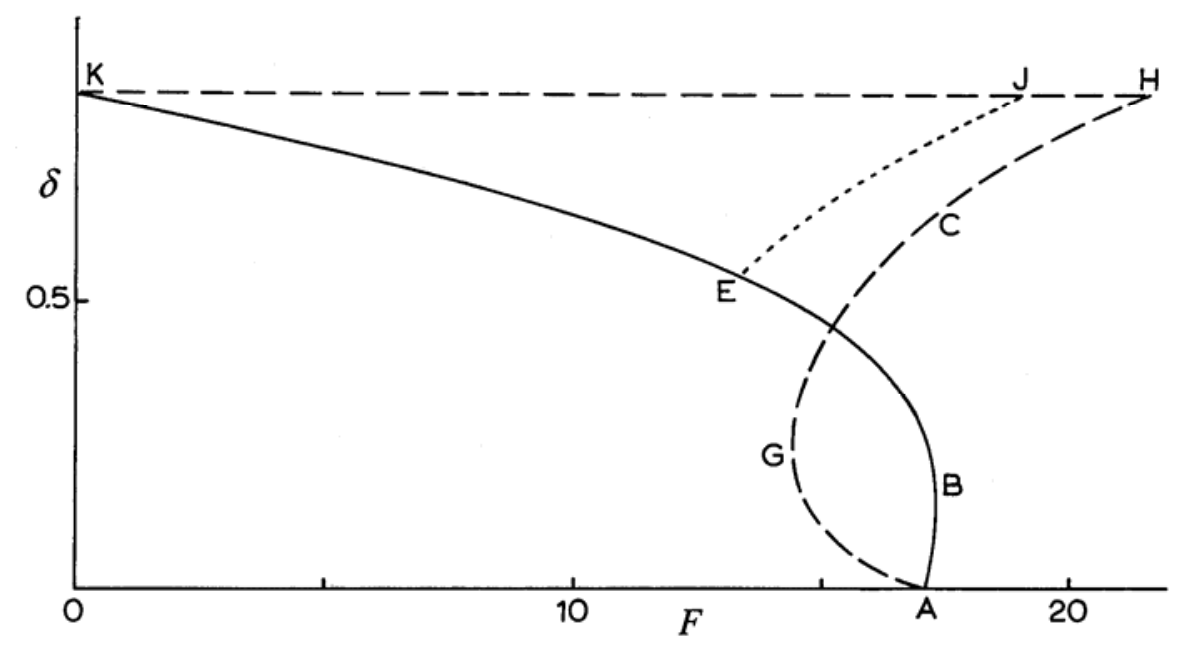

Fig. 2. Force-displacement curves for the frame with hinged joints $(k=0, b=1)$. Solid line: symmetric buckling, dashed line: asymmetric buckling, dotted line: secondary asymmetric buckling. 


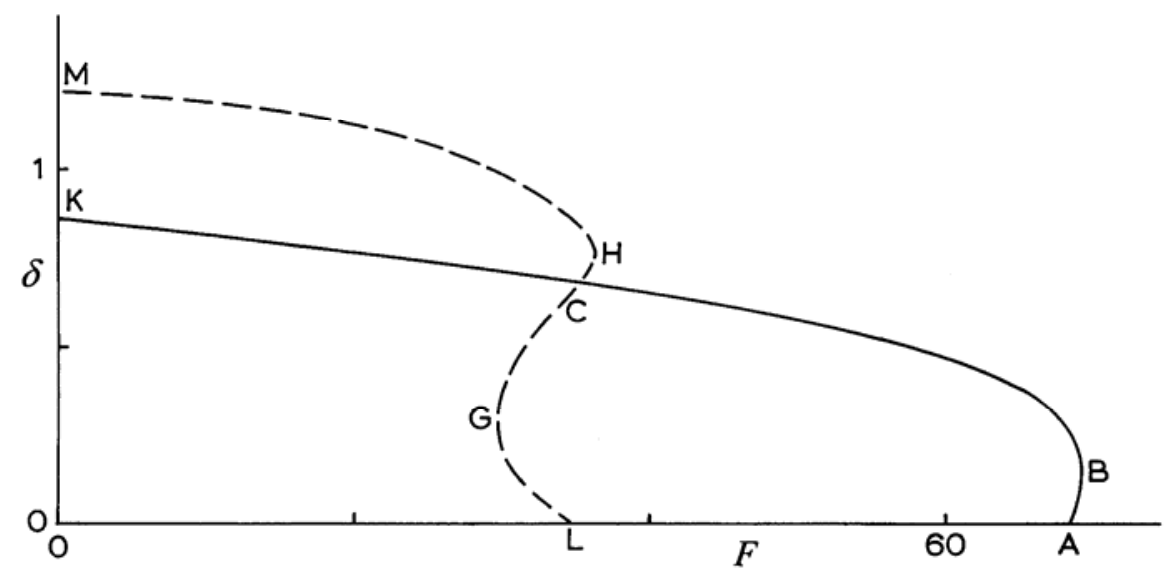

Fig. 3 Force-displacement curves for the frame with rigid joints $(k=\infty, b=1)$. Solid line: symmetric buckling, dashed line: asymmetric buckling. 


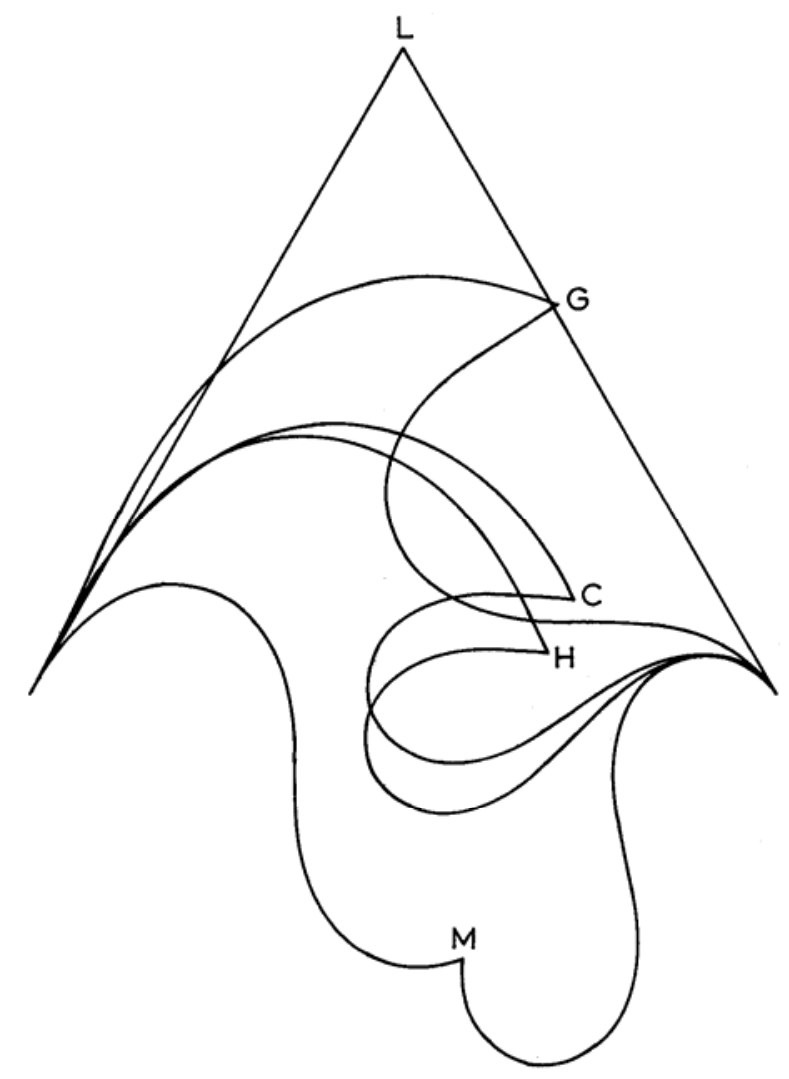

Fig. 4. Asymmetric deformed shapes of the rigidly-joined equilateral frame. Letters correspond to states in Fig. 3. 


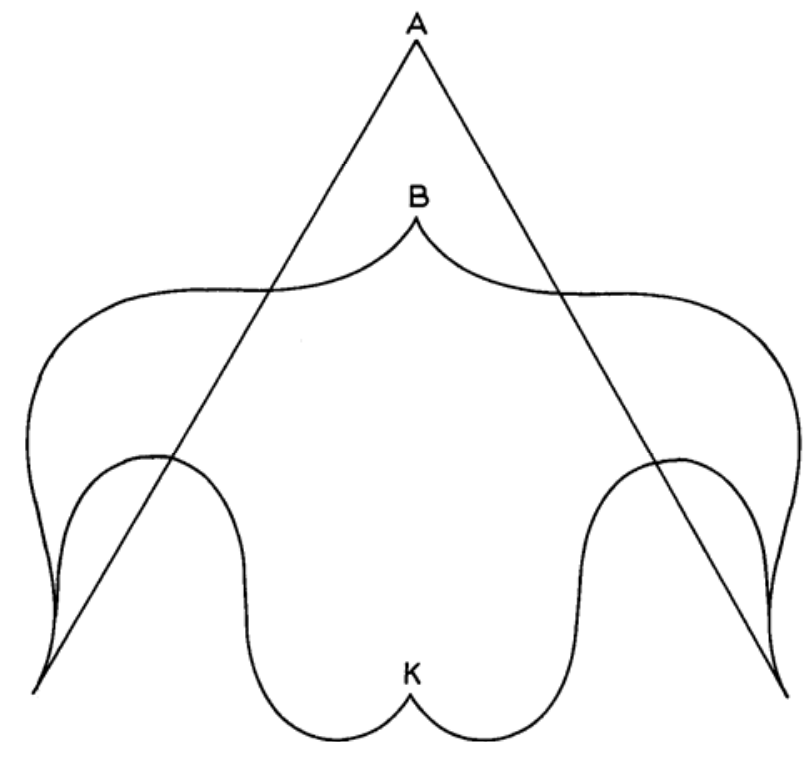

Fig. 5. Symmetric deformed shapes of the rigidly-joined equilateral frame. Letters correspond to states in Fig. 3. 


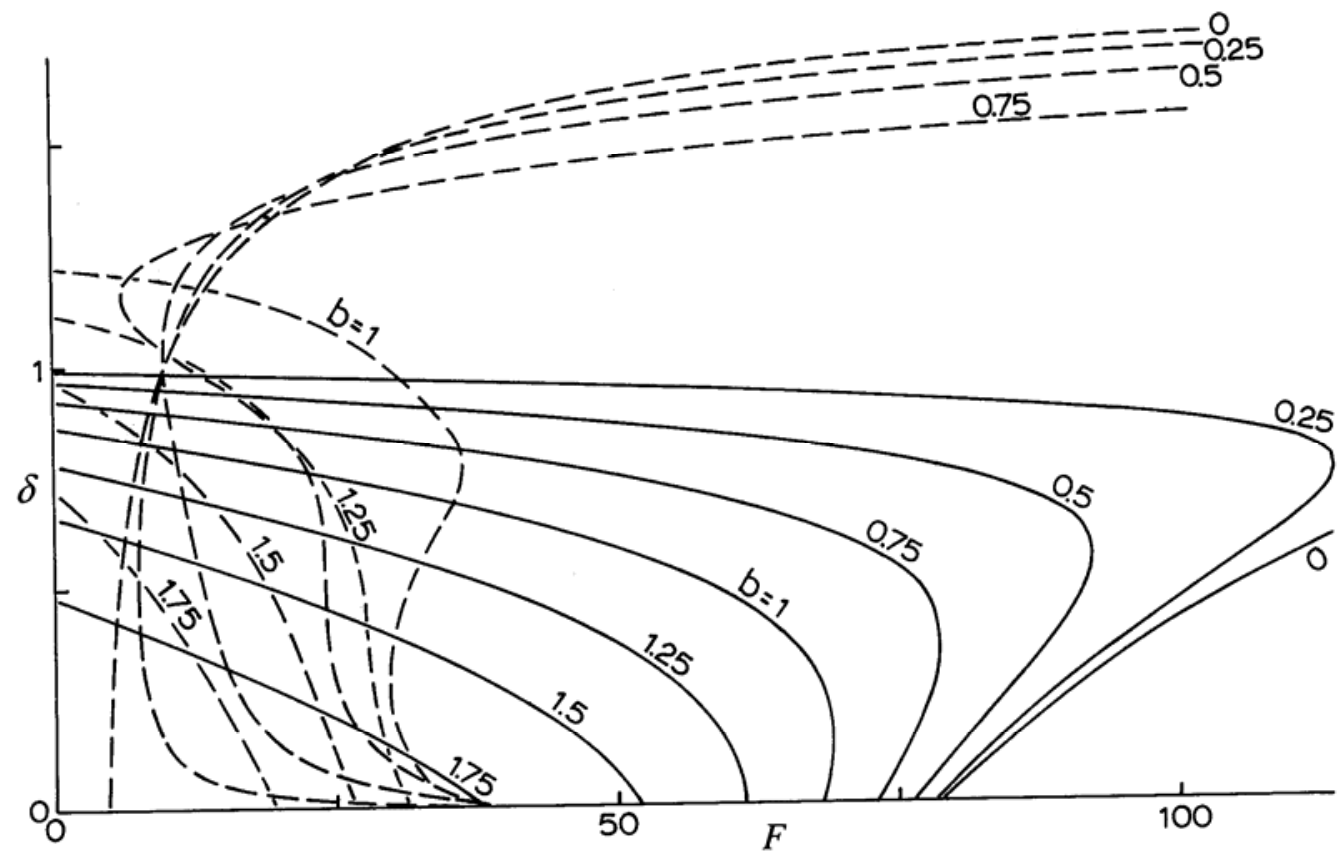

Fig. 6. Force displacement curves for the rigidly-joined frame with various base lengths b. Solid line: symmetric buckling, dashed line: asymmetric buckling. 


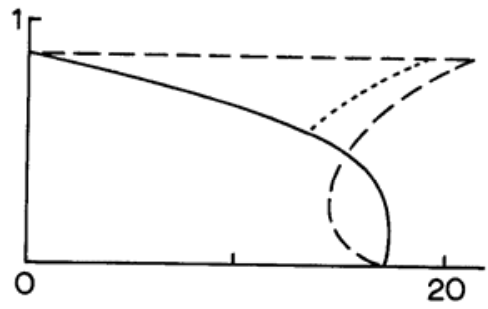

(a)

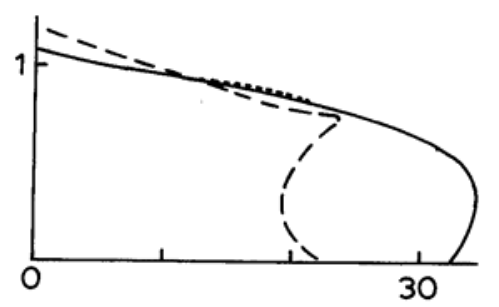

(c)

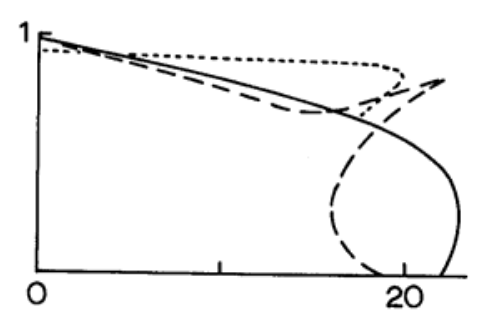

(b)

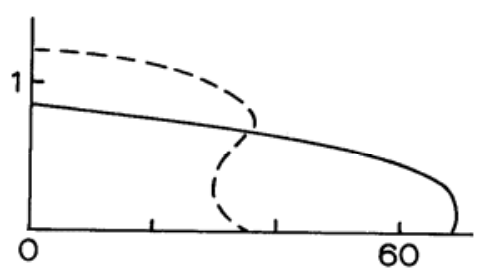

(d)

Fig. 7. Force displacement curves for $b=1$. (a) $k=0$, (b) $k=0.5$, (c) $k=2.0$, (d) $k=\infty$. 


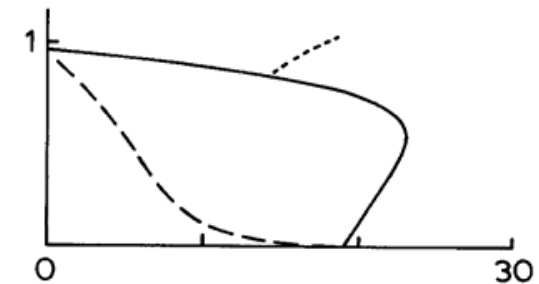

(a)

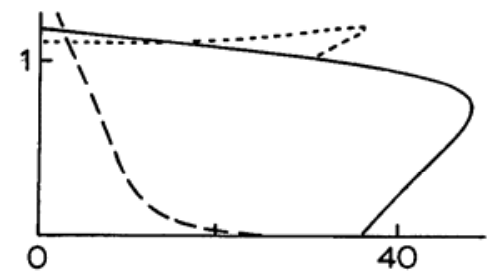

(c)

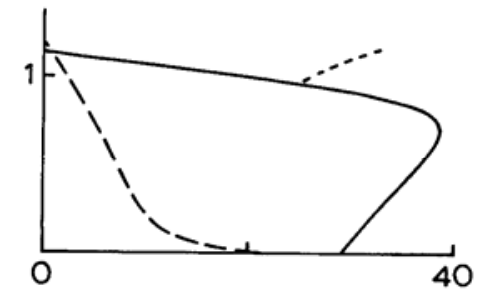

(b)

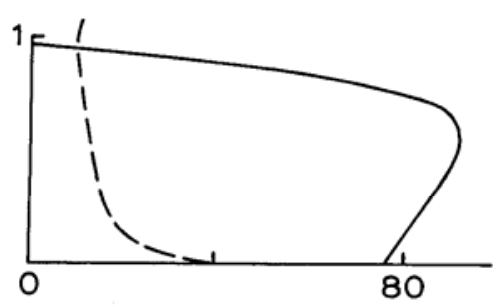

(d)

Fig. 8. Force displacement curves for $b=0.5$. (a) $k=0$, (b) $k=1.0$, (c) $k=2.0$, (d) $k=\infty$. 


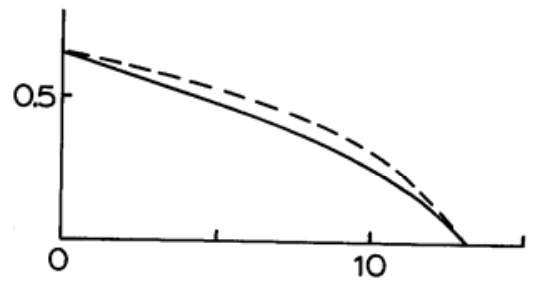

(a)

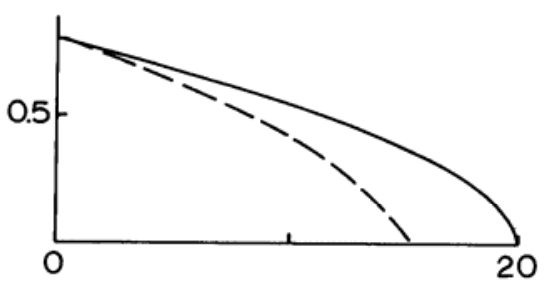

(c)

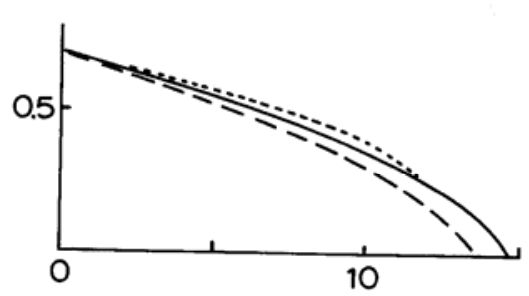

(b)

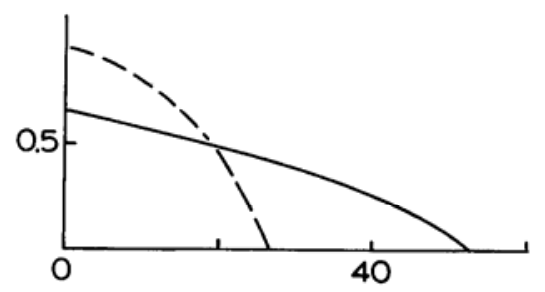

(d)

Fig. 9. Force displacement curves for $b=1.5$. (a) $k=0$, (b) $k=0.2$, (c) $k=1.0$, (d) $k=\infty$. 